\title{
Targeting SOD1 induces synthetic lethal killing in BLM- and CHEK2-deficient colorectal cancer cells
}

\author{
Babu V. Sajesh ${ }^{1,2}$, Kirk J. McManus ${ }^{1,2}$ \\ ${ }^{1}$ Department of Biochemistry and Medical Genetics, University of Manitoba, Winnipeg, Manitoba, Canada \\ ${ }^{2}$ Research Institute of Oncology and Hematology, Winnipeg, Manitoba, Canada \\ Correspondence to: \\ Kirk J. McManus, e-mail: Kirk.McManus@umanitoba.ca \\ Keywords: BLM, CHEK2, SOD1, synthetic lethality, drug targeting
}

Received: June 01, $2015 \quad$ Accepted: July 21, 2015

Published: July 31, 2015

\section{ABSTRACT}

Cancer is a major cause of death throughout the world, and there is a large need for better and more personalized approaches to combat the disease. Over the past decade, synthetic lethal approaches have been developed that are designed to exploit the aberrant molecular origins (i.e. defective genes) that underlie tumorigenesis. BLM and CHEK2 are two evolutionarily conserved genes that are somatically altered in a number of tumor types. Both proteins normally function in preserving genome stability through facilitating the accurate repair of DNA double strand breaks. Thus, uncovering synthetic lethal interactors of BLM and CHEK2 will identify novel candidate drug targets and lead chemical compounds. Here we identify an evolutionarily conserved synthetic lethal interaction between SOD1 and both BLM and CHEK2 in two distinct cell models. Using quantitative imaging microscopy, real-time cellular analyses, colony formation and tumor spheroid models we show that SOD1 silencing and inhibition (ATTM and LCS-1 treatments), or the induction of reactive oxygen species (2ME2 treatment) induces selective killing within BLM- and CHEK2-deficient cells relative to controls. We further show that increases in reactive oxygen species follow SOD1 silencing and inhibition that are associated with the persistence of DNA double strand breaks, and increases in apoptosis. Collectively, these data identify SOD1 as a novel candidate drug target in BLM and CHEK2 cancer contexts, and further suggest that 2ME2, ATTM and LCS-1 are lead therapeutic compounds warranting further pre-clinical study.

\section{INTRODUCTION}

Colorectal cancer (CRC) is a major cause of cancer-related deaths worldwide. In 2015, the American Cancer Society estimates that $\sim 132,700$ Americans will be newly diagnosed and $\sim 50,000$ additional individuals will succumb to the disease in 2015 [1]. These statistics highlight the need for novel personalized therapeutic strategies designed to better combat the disease. Synthetic lethality is one such strategy, and is defined as the lethal combination of two independently viable mutations. In a cancer context, a cancer-driving mutation is leveraged to lethality through the down-regulation (i.e. silencing or inhibition) of a synthetic lethal (SL) interactor (i.e. drug target) [2]. Thus, synthetic lethality exploits the causative genetic aberrations synonymous with tumor development and progression. Accordingly, identifying SL interactors of genes somatically altered in cancer will uncover novel candidate drug targets whose inhibitors represent lead therapeutic agents.

Recent gene re-sequencing efforts have uncovered a myriad of somatic mutations and deletions in genes associated with DNA double strand break (DSB) repair pathways [3, 4]. Somatic alterations of these cancer genes are correlated with genome instability (i.e. DNA damage and chromosome instability) [5], which contributes to the acquisition of subsequent mutations, some of which confer growth advantages that can enhance tumor growth and metastasis. For example, BLM and CHEK2 are somatically altered in a number of tumor types including CRC $[3,4,6]$, and normally function within the homology directed repair (HDR) pathway ("error-proof” DSB repair pathway). More specifically, BLM is a member of the RECQ helicase family, and harbors ATP-dependent 3'-5' 
DNA helicase activity (reviewed in [7]), which is required for HDR [7-12]. In addition, germline mutations in BLM are pathogenic for Bloom syndrome, an inherited disorder associated with an increased predisposition to develop many tumor types including CRC [13]. CHEK2 is a tumor suppressor that regulates genome stability [14]. It normally functions in HDR by inducing cell cycle checkpoints so that DSBs can be accurately repaired [15-18]. Thus, aberrant CHEK2 activity is associated with checkpoint defects, inadequate DNA repair, and cancer development. Accordingly, identifying novel strategies and candidate drug targets capable of exploiting genetic defects in $B L M$ and $C H E K 2$ are highly warranted.

In this study, we couple siRNA-based silencing and chemical compounds with semi-quantitative imaging microscopy, real time cellular analyses (RTCA), and biochemical assays to show that BLM and CHEK2 are SL with SOD1. We demonstrate that BLM- and CHEK2deficient CRC cells are selectively killed following SOD1 silencing and recapitulate these findings within an additional and unrelated cellular content. We further show that two SOD1 inhibitors (ammonium tetrathiomolybdate [ATTM] and Lung Cancer Screen-1 [LCS-1]) and one chemical mimetic (2-methoxyestradiol [2ME2]) phenocopy the SOD1 silencing results by inducing preferential killing within BLM- and CHEK2deficient cells. Using semi-quantitative microscopy and RTCA, we show that all three chemicals induce reactive oxygen species (ROS), promote persistent DNA DSBs and potentiate cellular cytotoxicity through an apoptotic mechanism. Finally, we show that drug treatments significantly decrease the number and size of $B L M$ and CHEK2-deficient cells in 2D colony and 3D tumor spheroid formation assays, respectively. Collectively, our data shows that BLM and CHEK2 are SL with SOD1, and further identify 2ME2, ATTM and LCS-1 as lead candidate compounds warranting further pre-clinical study.

\section{MATERIALS AND METHODS}

\section{Cell culture}

HCT116 (BLM-proficient and CHEK2-proficient) cells were purchased from American Type Culture Collection. BLM-deficient and CHEK2-deficient, HCT116 cells were generously provided by Dr. Bert Vogelstein (Johns Hopkins University, Baltimore). All HCT116 cells were grown in McCoy's 5A medium (HyClone) containing $10 \%$ fetal bovine serum. Immortalized (telomerase) BJ normal human skin fibroblasts, hTERT, were generously provided by C.P. Case [19] (University of Bristol, Bristol, UK) and were grown in DMEM supplemented with $10 \%$ fetal bovine serum. Cells were authenticated based on the recovery, viability, growth and morphology, while parental HCT116 and hTERT cells were also authenticated by spectral karyotyping as detailed elsewhere [20]. BLM and CHEK2 expression was confirmed in all cell lines by Western blots. All cells were grown in a $37^{\circ} \mathrm{C}$ humidified incubator with $5 \% \mathrm{CO}_{2}$.

\section{Gene silencing}

Cells were transiently transfected with siRNA duplexes using RNAiMax (Invitrogen) as described [20]. ON-TARGETplus (Dharmacon) siRNA duplexes targeting SOD1, BLM, CHEK2, GAPDH and PLK1 were employed as either individual duplexes or pools (four distinct duplexes targeting the gene of interest), as detailed previously [20]. Gene silencing was confirmed by Western blots using the antibodies and dilutions indicated in Supplementary Table 1.

\section{Direct SL tests}

High-content microscopy was used to evaluate the SL interactions as detailed elsewhere [20]. Briefly, 8,000 BLM-deficient, CHEK2-deficient and control (HCT116) cells, and 4,000 hTERT cells were automatically dispensed into each well of a 96-well plate (BioTek; EL406). Cells were transfected in sextuplet (i.e. 6-wells) with either individual or pooled siRNAs targeting BLM, CHEK2, SOD1, and controls (GAPDH and PLK1) as described [20]. Cells were permitted to grow for 4- (HCT116) or 5 -days (hTERT) following which cells were fixed (4\% paraformaldehyde), and counterstained with Hoechst 33342 (Sigma; $0.2 \mu \mathrm{M}$ ). Images were acquired using a Cytation 3 (BioTek) equipped with a $10 \times$ objective $(0.3$ numerical aperture), a 16-bit gray scale charged couple device camera, and GEN5 software. Twelve central and non-overlapping images were acquired per well (condition), and the total number of cells in each well and condition were determined. All data were imported into Prism v6.0 (GraphPad), normalized to GAPDH silenced controls, and basic statistical analyses (e.g. mean, standard deviation, Student's $t$-tests, etc.) were performed. To address reproducibility all experiments were conducted a minimum of 3-times.

\section{Dose response curves}

Standard dose response curves were generated as detailed previously [20] using a 10-fold (100pM to $1 \mathrm{M}$ ) serial dilution for 2ME2, ATTM, or LCS-1 [21]. Briefly, $\sim 8,000$ cells were seeded into each well of a 96-well plate, permitted to attach, and growth medium supplemented with appropriate concentrations of $2 \mathrm{ME} 2$, ATTM, LCS-1 or vehicle control (DMSO) were added to wells in sextuplet. Following 3-days of growth, cells were fixed, counterstained, imaged and analyzed as above. All data were normalized to DMSO-treated controls and half maximal effective concentrations $\left(\mathrm{EC}_{50}\right)$ values were determined. The $\mathrm{EC}_{50}$ values calculated from the 
BLM- and CHEK2-deficient cells were employed in all subsequent experiments.

\section{ROS detection}

ROS were detected using the Image-IT LIVE Green ROS detection kit (Molecular Probes) as detailed elsewhere [20] with minor modifications. Briefly, 8,000 cells were seeded into each well of a 96-well plate, and permitted to attach. The following day, SOD1 or GAPDH were silenced in control, BLM- and CHEK2-deficient cells for $48 \mathrm{~h}$ or cells were treated with compounds (2ME2, ATTM, LCS-1 and DMSO) for $6 \mathrm{~h}$. Each condition was performed in sextuplet and repeated two additional times. Images were acquired (Cytation 3), signal intensities were determined from raw, unprocessed images (GEN5), and semi-quantitative analyses were performed as described $[20,22]$. All data were imported into Prism, normalized to untreated controls, and basic statistical analyses (e.g. mean, standard deviation, Student's $t$-tests, etc.) were performed.

\section{Quantitative imaging microscopy}

Semi-quantitative imaging microscopy was employed to evaluate the presence of DNA DSBs as represented by the total signal intensities of two surrogate markers, namely $\gamma-\mathrm{H} 2 \mathrm{AX}$ and 53BP1, as described previously [20, 22]. Briefly, 8, 000 cells were seeded into each well of a 96-well plate, permitted to attach, and grow for $48 \mathrm{~h}$. Next, cells were treated with Bleomycin $(0.1 \mu \mathrm{g} / \mathrm{mL})$ for $2 \mathrm{~h}$, DMSO for $42 \mathrm{~h}$, or $2 \mathrm{ME} 2$, ATTM and LCS-1 for $6 \mathrm{~h}$. The media from a subset of wells containing 2ME2, ATTM, and LCS-1 was aspirated, washed once with pre-warmed phosphate buffered saline ( $\mathrm{pH} 7.4$ ), replaced with fresh growth media lacking 2ME2, ATTM and LCS-1, and cells were permitted to grow for an additional $36 \mathrm{~h}$. Following washout and recovery, cells were processed for semi-quantitative microscopy as detailed [20], and images were acquired as described above (Direct SL Tests). Each condition was performed in sextuplet and repeated at least two additional times with $\gamma$-H2AX and 53BP1 signal intensities determined from raw, unprocessed images. Apoptosis was evaluated by quantifying the total signal intensity of cleaved Caspase 3 as described previously [20]. All data were imported into Prism and were normalized to the appropriate negative or vehicle control. Antibodies and working dilutions are presented in Supplementary Table 1.

\section{Real time cellular analyses}

RTCA were performed in quadruplicate and repeated twice using an xCELLigence RTCA-dual plate (DP) system (Acea Biosciences) as detailed [20]. Approximately 8,000 cells were seeded into each well of an E-Plate (Acea Biosciences) and growth (electrical impedance) was measured every 10 minutes. DMSO,
2ME2, ATTM, or LCS-1 were supplemented into growth medium when cells attained $\sim 25 \%$ of their untreated maximal values ( $\sim 8 \mathrm{~h}$ post seeding) and growth was monitored for up to 7-days. All data were imported into Prism and growth curves were plotted for each condition of treatment.

\section{ROS scavenging/chemical rescue}

$\mathrm{N}$-acetyl-L-cysteine (NAC) was used to scavenge excessive ROS. The maximum tolerated dose of NAC was determined for BLM-deficient $(2 \mu \mathrm{M})$ and CHEK2deficient $(5 \mu \mathrm{M})$ cells by RTCA. RTCA was performed as above, but with media supplemented with DMSO, 2ME2, ATTM or LCS-1 with or without NAC. All data were imported into Prism where growth curves were plotted.

\section{Colony formation and 3D tumor spheroid assays}

The effect of prolonged DMSO, 2ME2, ATTM and LCS-1 treatments was determined using a standard 28-day colony forming assay as described [20], and tumor spheroid assays as directed by the manufacturer (InSphero). In brief, 1,000 BLM- or CHEK2-deficient, or control cells were dispensed into GravityPLUS plates, and tumors were permitted to form for 2-days, whereupon they were released into GravityTRAP plates and cultured in growth medium containing DMSO, 2ME2, ATTM, or LCS-1. Media containing DMSO, 2ME2, ATTM, or LCS-1 were replaced every 2- to 3-days. Cells were incubated for 14-days, at which point nuclei were counterstained with Hoechst 33342, and the medial plane of each tumor spheroid was imaged using a Cytation 3 $(10 \times$ objective; NA $=0.13)$. ImageJ was employed to determine the maximal diameter of the spheroid [23]. All data were imported into Prism, normalized to the appropriate DMSO-treated control, and Student's $t$-tests were performed. Finally, all experiments were performed in sextuplet and repeated at least two additional times.

\section{RESULTS}

\section{BLM and CHEK2 are synthetic lethal with SOD1}

To identify novel and lead candidate drug targets to evaluate in a CRC context, we recently employed a cross-species candidate gene approach and identified the SL interactors of yeast genes whose human orthologs are somatically altered in CRC. We queried BioGRID [24] and identified all SL interactions for the 692 yeast chromosome instability genes [20]. As an entry point, two-dimensional hierarchical clustering was performed [20], and a collection of 30 genes each harboring $>22$ SL interactors were identified. This collection was highly 
enriched for genes encoding functions within DSB repair, particularly HDR including $s g s 1$ and $d u n 1$, which are the yeast orthologs of human $B L M$ and $C H E K 2$, respectively. Interestingly, $s g s 1$ and $d u n 1$ are SL with several members of the evolutionarily conserved superoxide dismutase pathway, including superoxide dismutase-1 (yeast sod1/ human SOD1). This pathway normally functions to remove excess ROS through a two-step process initially regulated by SOD1, which requires $\mathrm{Cu}^{2+}$ as an essential co-factor $[25,26]$. Excessive ROS induce a variety of cellular damage including DSBs, which we presumed would not be accurately repaired in HDR-defective cells (e.g. BLM and CHEK2-deficient), and would underlie cell cytotoxicity. Accordingly, SOD1 represents a strong candidate to pursue in a cross-species approach designed to identify novel drug targets (i.e. SL interactors) for $B L M$ and CHEK2.

In budding yeast, sgs 1 and dun 1 are SL with sod1 [27]. To determine whether SOD1 is SL with BLM and $C H E K 2$ in humans, we employed an established siRNA-based approach [20, 28]. Briefly, BLM-deficient (or CHEK2-deficient) and control HCT116 (BLM- and CHEK2-proficient) cells are silenced with either individual or pooled siRNAs targeting SOD1 and controls (GAPDH), and the total number of cells remaining is statistically compared. A SL interaction is expected to result in fewer cells within the deficient cell lines. Before performing the SL tests, we first confirmed BLM and CHEK2 expression levels within all cell lines by Western blots (Supplementary Figure 1), and evaluated the silencing efficiency of two individual (siSOD1-2 and siSOD1-3) and pooled (siSOD1-P) siRNA duplexes (Figure 1A). Next, we performed direct SL tests and as predicted there were visually striking decreases in the number of $B L M$ and $C H E K 2$-deficient cells relative to controls (Figure 1B) that quantitative imaging microscopy determined to be statistically significant (Figure 1C and Supplementary Table 2). Further scrutiny of the images revealed a population of BLM- and CHEK2-deficient cells exhibiting hallmarks of apoptosis including nuclear blebbing and increased chromatin condensation that were not readily apparent within the controls (Figure 1B). Collectively, these data suggest that SOD1 silencing selectively induces cytotoxicity within BLM- and CHEK2-deficient cells, and further suggest that these genetic interactions are evolutionarily conserved.

Although the above observations suggest $B L M$ and $C H E K 2$ are SL with SOD1, it remains possible that the putative interactions are due to background mutations that accrued during the generation of the BLM- and CHEK2-deficient cells. To assuage this possibility, dual siRNA experiments (e.g. BLM plus SOD1 silencing) were performed within the parental, HCT116 cells. In agreement with the above data, Figure 1D shows that the simultaneous silencing of either $B L M$ or $C H E K 2$ with $S O D 1$ resulted in fewer cells than each condition alone, or the expected number as calculated by a multiplicative model (Supplementary Table 3). The percentage of cells remaining was similar and $\sim 60 \%$ with either the individual or pooled approaches for both BLM and CHEK2. Although the total decrease in cell numbers was not as large as with the BLM- and CHEK2-deficient cells employed above, we attribute this to the residual proteins remaining following siRNA-based silencing compared with the their complete absence within the BLM- and CHEK2-deficient cells (Supplementary Figure 2).

To extend these findings beyond the HCT116 context employed above, analogous dual siRNA-based experiments were performed in hTERT, a karyotypically stable and immortalized fibroblast cell line. Western blots were first performed to confirm silencing efficiencies (Supplementary Figure 3), and in agreement with the HCT116 findings, dual siRNA-based silencing (siBLM/siCHEK2 and siSOD1) resulted in fewer hTERT cells than expected using a multiplicative model (Supplementary Figure 4 and Supplementary Table 4). Collectively, these data support the evolutionarily conserved SL interaction between $B L M$ or $C H E K 2$ and $S O D 1$, and further demonstrate that these interactions are independent of cell type.

\section{ME2, ATTM and LCS-1 induce selective Killing in BLM- and CHEK2-deficient cells in a ROS-dependent manner}

Having established that $B L M$ and $C H E K 2$ are SL with SOD1, we now wished to determine if three chemical compounds, 2ME2, ATTM and LCS-1 predicted to phenocopy SOD1 silencing could substitute for the siRNAs and induce death specifically within the BLMand CHEK2-deficient cells. While 2ME2 induces ROS including superoxide anions [29], ATTM and LCS-1 are a $\mathrm{Cu}^{2+}$ chelator [26] (required for SOD1 activity) and a SOD1 inhibitor [21] respectively. Prior to evaluating the SL interactions, standard dose response curves were generated for each compound within the BLM- and CHEK2-deficient, and control cells. As predicted, the $B L M$ - and CHEK2-deficient cell lines are hypersensitive to each compound relative to controls (Figure 2). In addition, increases in cells exhibiting apoptotic hallmarks were also observed within the BLM- and CHEK2-deficient cells following treatments. Table 1 presents the $\mathrm{EC}_{50}$ (effective concentration at which $50 \%$ of the cells remain relative to DMSO-treated controls) values calculated for the $B L M$ - and CHEK2-deficient cells relative to controls. In support of chemogenetic SL interactions, the $\mathrm{EC}_{50}$ values for 2ME2, ATTM and LCS-1 are approximately 193-, 54- and 3, 150-fold lower, respectively, in BLM-deficient cells, while they are approximately 1,230-, 1,175- and 1,892-fold lower in CHEK2-deficient cells, respectively. Finally, a fluorogenic ROS indicator coupled with semiquantitative imaging microscopy and was employed to confirm that 2ME2, ATTM and LCS-1 treatments each 
A
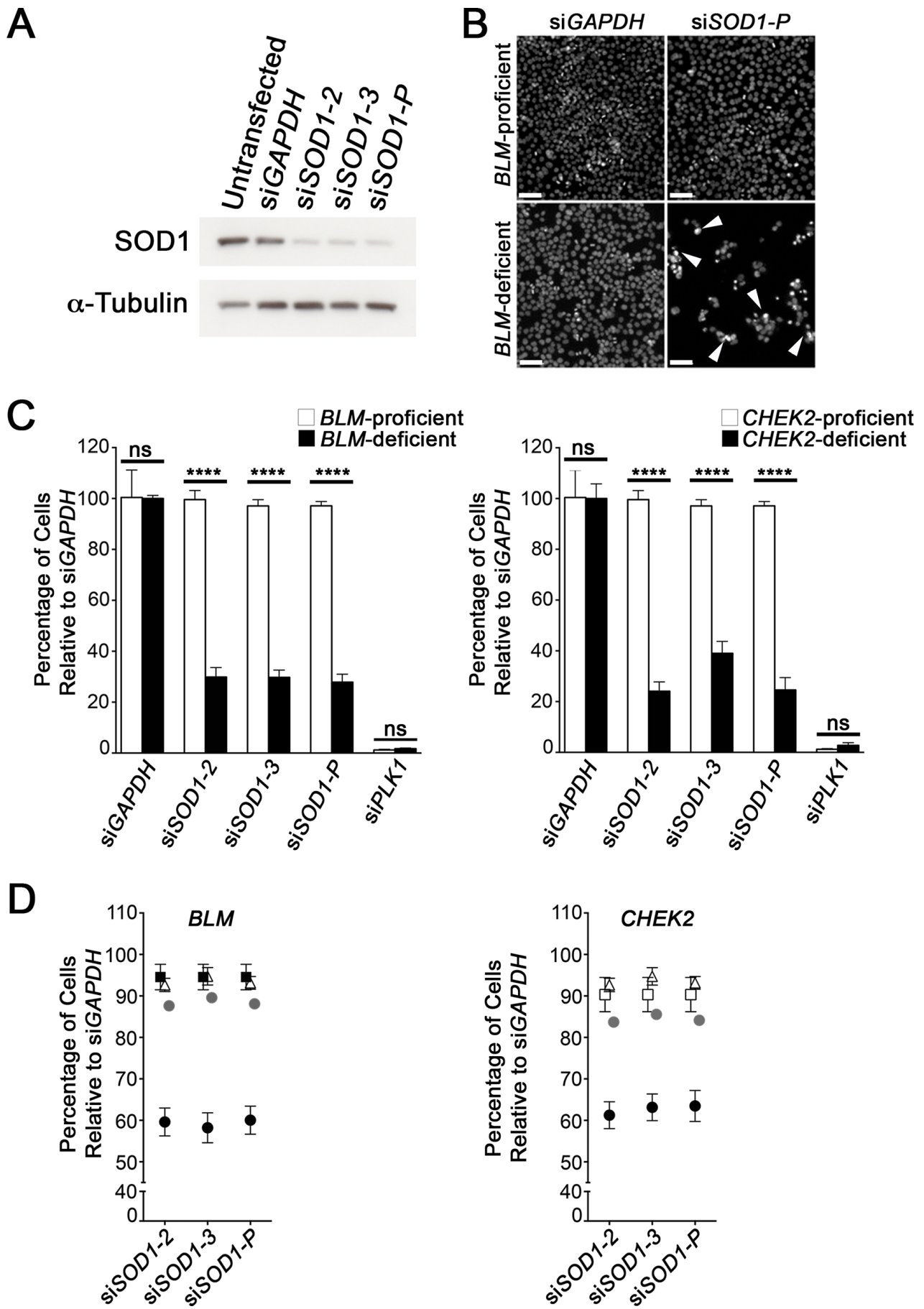

Figure 1: BLM and $C H E K 2$ are synthetic lethal with SOD1. A. Western blot depicting SOD1 silencing in HCT116 cells with either individual (siSOD1-2 and siSOD1-3) or pooled (siSOD1-P) siRNA duplexes relative to controls (untransfected and siGAPDH); $\alpha$-Tubulin serves as a loading control. B. Representative low-resolution images depicting the decrease in Hoechst stained nuclei (bottom right quadrant) following SOD1 silencing in $B L M$-deficient cells. Arrowheads identify nuclei exhibiting apoptotic hallmarks. Scale bars represent $100 \mu \mathrm{m}$. C. Graph depicting the statistically significant decrease in BLM- (left) or CHEK2-deficient cells (right) following SOD1 silencing relative to controls. The statistical significance is indicated ( $n s$, not significant; ****, $p$-value $<0.0001$ ). GAPDH serves as the negative control, while $P L K 1$ is an essential gene used as a positive control for death and a transfection indicator. D. Graphs depicting the SL interaction observed following simultaneous silencing of BLM (left) or CHEK2 (right) with SOD1 in HCT116 cells. Presented are the mean normalized percentages $( \pm \mathrm{SD})$ for the individual silencing of either BLM (solid squares) or CHEK2 (open squares) and SOD1 (open triangles), and the expected value (grey circles) determined for the dual combined siRNAs as calculated using a multiplicative model. Solid circles identify the actual observed values for the simultaneous dual silencing (i.e. BLM and SOD1, or CHEK2 and SOD1) and are lower than the expected values indicating a SL phenotype. 
A

BLM-proficient

- BLM-deficient

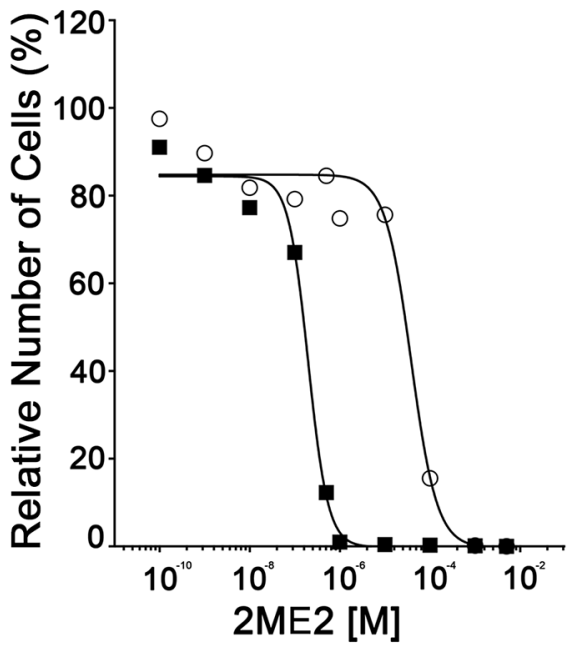

CHEK2-proficient

$\triangle$ CHEK2-deficient

B
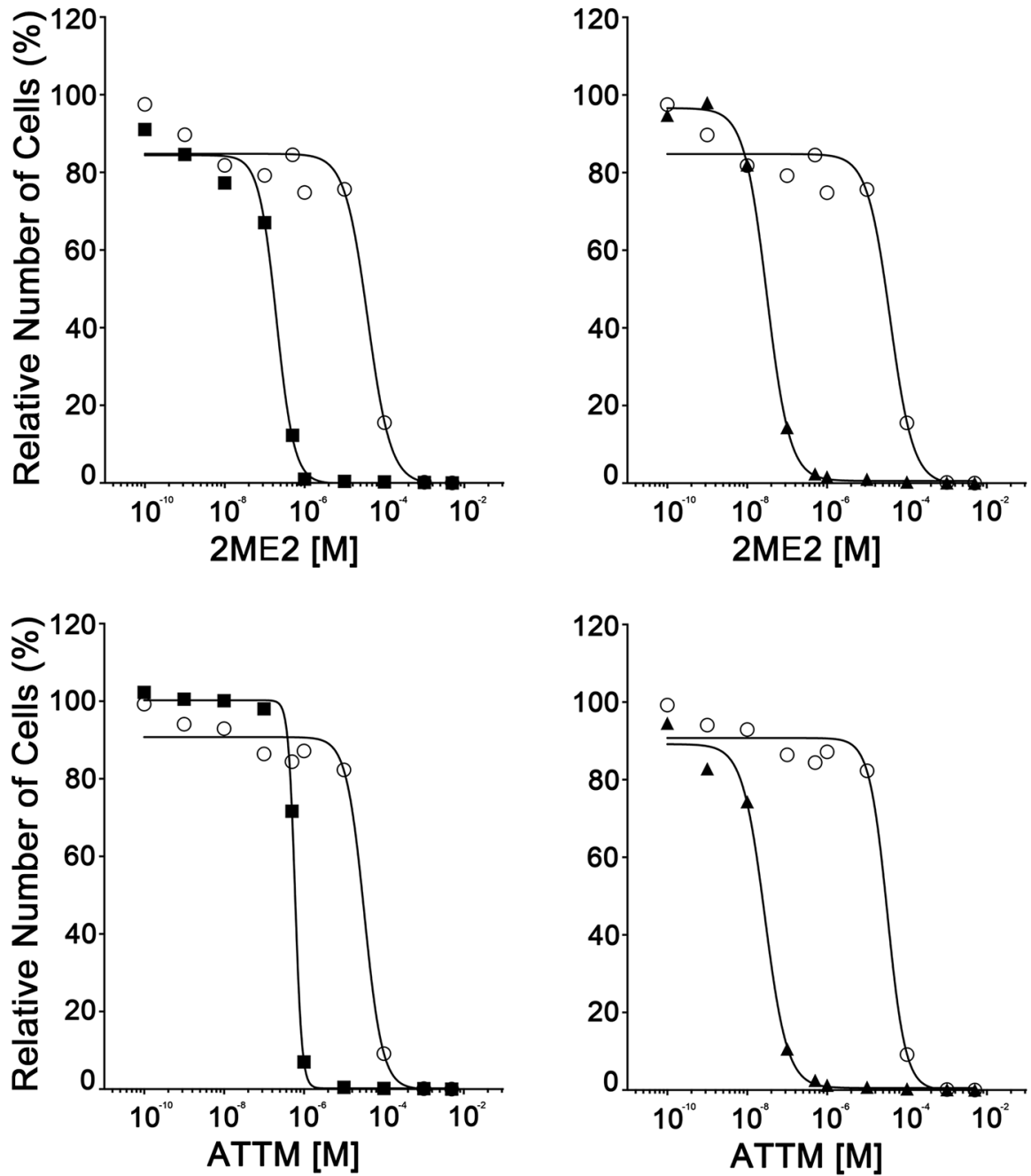

C
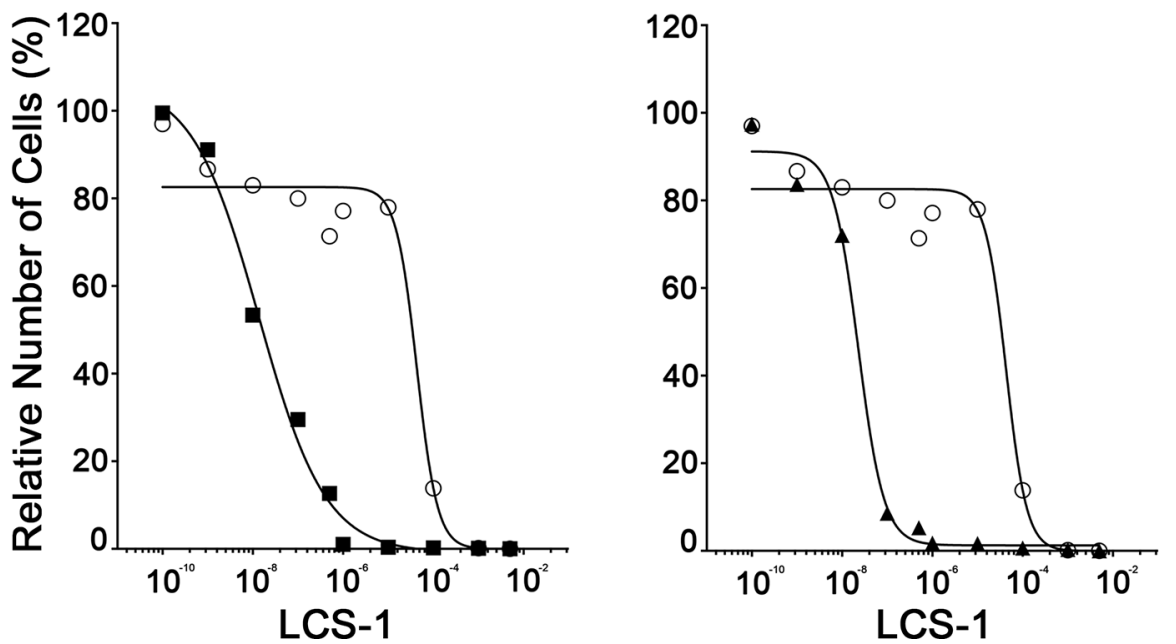

Figure 2: BLM- and $C H E K 2$-deficient cells are hypersensitive to 2ME2, ATTM and LCS-1. Standard dose response curves for cells (indicated at top) treated with varying concentrations of $2 \mathrm{ME} 2$ A. ATTM B. and LCS-1 C. Data are normalized to the respective DMSO-treated controls. 
Table 1: $\mathrm{EC}_{50}$ values calculated from dose response curves

\begin{tabular}{|l|c|c|c|}
\hline Compound & HCT116 (control) $\mathbf{E C}_{\mathbf{5 0}}(\mathbf{n M})$ & BLM-deficient EC $_{\mathbf{5 0}}(\mathbf{n M}) / \mathbf{F I}^{\mathbf{A}}$ & CHEK2-deficient EC $_{\mathbf{5 0}}(\mathbf{n M}) / \mathbf{F I}^{\mathbf{A}}$ \\
\hline 2ME2 & $3.82 \times 10^{4}$ & $1.98 \times 10^{2} / 193$ & $3.10 \times 10^{1 / 1232}$ \\
\hline ATTM & $3.23 \times 10^{4}$ & $5.98 \times 10^{2} / 54$ & $2.75 \times 10^{1 / 1175}$ \\
\hline LCS-1 & $4.31 \times 10^{4}$ & $1.37 \times 10^{1} / 3145$ & $2.28 \times 10^{1} / 1889$ \\
\hline
\end{tabular}

${ }^{\mathrm{A}} \mathrm{FI}$; Fold increase in sensitivity relative to the corresponding HCT116 control.

induce ROS formation (Supplementary Figure 5), which also occurs following SOD1 silencing (Supplementary Figure 6).

To determine if the diminished cell numbers were due to cell cycle arrest or cellular cytotoxicity RTCA (i.e. growth curves) were performed. RTCA employs electrical impedance as a measure of cellular proliferation and can easily discern altered growth rates from cell cycle arrests and cellular cytotoxicity. To restrict the therapeutic effect to BLM- and CHEK2-deficient cells, we specifically employed the $\mathrm{EC}_{50}$ values determined for those cells. As predicted, the growth curves generated for the control cells (Figure 3A) were virtually indistinguishable irrespective of treatment (e.g. 2ME2, ATTM, LCS-1 and DMSO). However, there was considerable variation in the growth curves generated within the BLM- and CHEK2deficient cells (Figure 3A), with each showing a rapid decline in cell index that is indicative of cell cytotoxicity. More specifically, within the $B L M$-deficient cells, $2 \mathrm{ME} 2$ and LCS-1 gave superimposable growth curves with a cell index marginally lower ( 0.5 a.u.) than that of DMSO treated controls, whereas ATTM treated cells had an increase in cell index (1.4 a.u.) approximately 1-day post-treatment. Approximately 2-days post-treatments, there was a rapid decline in the cell index in each of the condition of treatment that was not readily apparent in the DMSO treated controls. On the other hand, within the CHEK2-deficient cells, 2ME2, ATTM and LCS-1 treated cells showed a markedly high cell index $(1.2,5$ and 3 a.u., respectively) when compared to DMSO treated controls in 24 hours of treatment with the chemical compounds, which is likely due to a temporary cell cycle arrest and flattening of the cell body (unpublished observation) causing an increase in cellular contacts and thus electrical impedance. Following a temporary arrest as evidenced by a plateau in cell index, there was a rapid and sharp decline in cell index that occurred approximately 2-days (2ME2), 3-days (LCS-1), or 4-days (ATTM) posttreatment. The rapid declines in cell indices for $B L M$ and CHEK2-deficient cells treated with 2ME2, ATTM and LCS-1 are indicative of strong cytotoxic effects, and are in agreement with the images exhibiting apoptotic hallmarks (see Figure 1B). These data further show that 2ME2, ATTM and LCS-1 have minimal impact on the growth of control cells indicating that the compounds and concentrations employed are selective to the BLM- and CHEK2-deficient cells.
To determine if oxidative stress (i.e. ROS generation) was required to induce the cytotoxicity observed above, NAC, an established ROS scavenger was employed in phenotypic rescue experiments. In all instances, NAC addition was sufficient to restore the growth of the BLM- and CHEK2-deficient cells treated with 2ME2, ATTM and LCS-1 to those of the vehicle control (Figure 3B). Collectively, these data indicate that 2ME2, ATTM and LCS-1 induce preferential cytotoxicity in the BLM- and CHEK2-deficient cells, and further show that ROS contributes to the cytotoxic effect.

\section{ME2, ATTM and LCS-1 induce persistent DNA DSBs in BLM- and CHEK2-deficient cells}

We now sought to determine the underlying mechanism contributing to the increases in cytotoxicity observed within the BLM- and CHEK2-deficient cells treated with 2ME2, ATTM and LCS-1. Since oxidative stress induces various types of cellular damage including DSBs [30-32], we reasoned that excessive DSBs caused by treatments would not be adequately repaired in HDR-defective cells, and would ultimately lead to cell cytotoxicity. Accordingly, the prevalence and persistence of DSBs was evaluated by semi-quantitative imaging microscopy at various time points using two wellestablished surrogate markers for DSBs, namely $\gamma$-H2AX and 53BP1. As expected, visually apparent and statistically significant increases in $\gamma-\mathrm{H} 2 \mathrm{AX}$ and 53BP1 signal intensities within BLM- (Figure 4), CHEK2-deficient (Supplementary Figure 7) and control cells treated with 2ME2, ATTM and LCS-1 for $6 \mathrm{~h}$ relative to DMSO treated controls. However, following compound washout and a 36 $\mathrm{h}$ recovery phase, $\gamma-\mathrm{H} 2 \mathrm{AX}$ and 53BP1 intensities remained elevated within the BLM- and CHEK2-deficient cells, but returned to basal levels within the controls. Thus, these data support the premise that 2ME2, ATTM and LCS-1 treatments induce ROS formation that promotes persistent DNA DSBs, which cannot be adequately repaired within the BLM- and CHEK2-deficient cells, and underlies the observed increases in cell cytotoxicity.

\section{ME2, ATTM and LCS-1 treatments induce apoptosis in BLM- and CHEK2-deficient cells}

Although the above data show that BLM- and CHEK2-deficient cells are hypersensitive to 2ME2, 

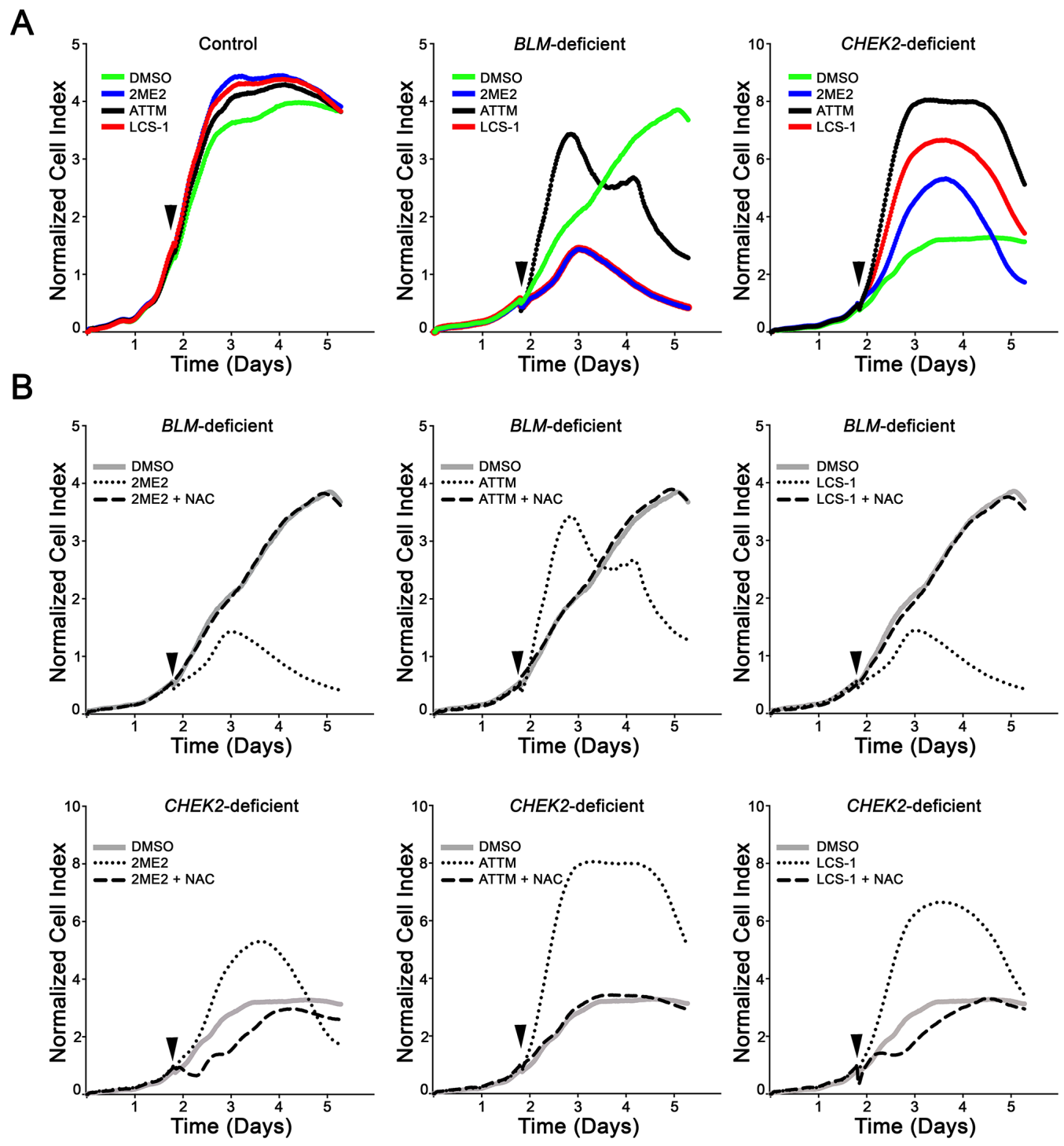

Figure 3: NAC administration rescues the hypersensitivity of $B L M$-deficient and $C H E K 2$-deficient cells to 2ME2, ATTM and LCS-1. A. RTCA growth curves for control (left), BLM- (middle) and CHEK2-deficient (right) cells treated with DMSO, 2ME2, ATTM or LCS-1. Arrowheads identify the timepoints at which the chemicals were administered. B. RTCA depicting NAC rescue of BLM- (top panels) or CHEK2-deficient cells (bottom panels) treated with 2ME2 (left), ATTM (middle) or LCS-1 (right). Arrowheads identify the timepoints at which the chemicals were administered. Note that NAC addition restores growth back to approximately that of the corresponding DMSO-treated controls for all three compounds and in both cell lines.

ATTM and LCS- 1 treatments, they do not address the underlying mechanism of death. However, recall that further scrutiny of images acquired following SOD1 silencing or compound treatments suggest apoptosis may be a contributing factor. Accordingly, we now wished to formally examine whether apoptosis contributes to the cytotoxicity observed within the BLM and CHEK2 chemogenetic interactions identified above. Using semiquantitative imaging microscopy and an antibody against cleaved Caspase 3, a key downstream apoptotic regulator, we evaluated apoptosis in cells treated with 2ME2, ATTM and LCS-1. As anticipated, BLM- and CHEK2-deficient cells treated with compounds exhibited statistically significant increases in the abundance of cleaved Caspase
3 relative to controls (Figure 5). More specifically, a 2.7to 3.0-fold increase in DMSO-normalized cleaved Caspase 3 signal intensities occurred within the BLM- and CHEK2deficient cells relative to controls. These data show that apoptosis contributes to the cytotoxicity observed following 2ME2, ATTM and LCS-1 treatments within the $B L M$ - and CHEK2-deficient cells.

\section{ME2, ATTM and LCS-1 impair growth of BLM- and CHEK2-deficient cells in 3D cultures}

To evaluate the long-term ( 2 and 4 week) effects 2ME2, ATTM and LCS-1 have on cellular growth both colony formation in soft agar and 3D tumor sphere assays 

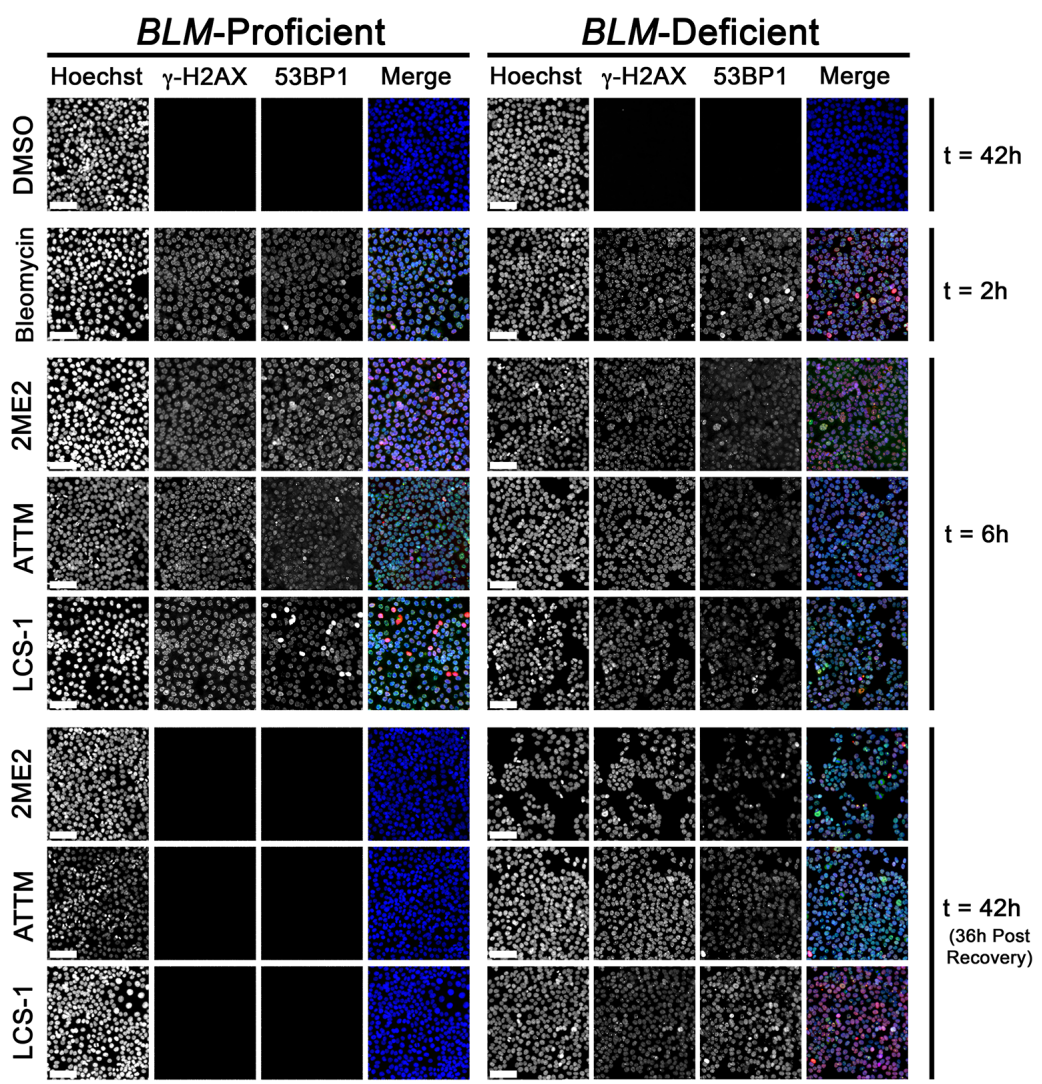

B

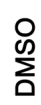

器
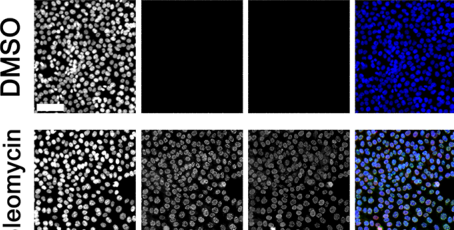

出
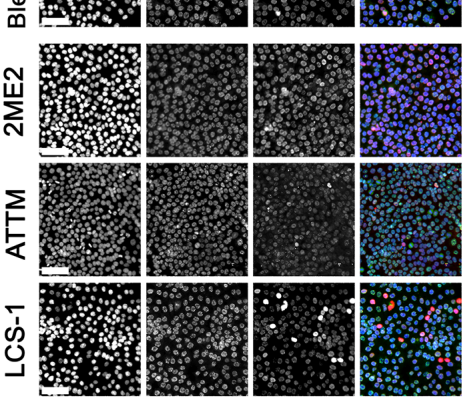

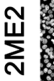
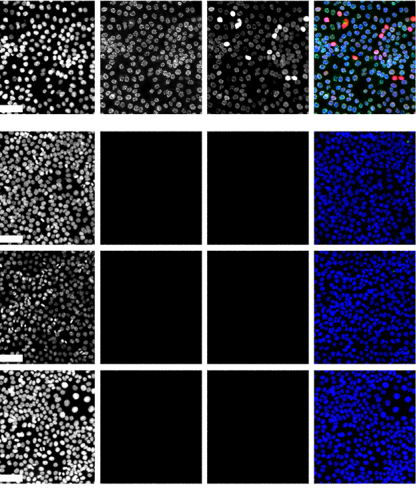

E

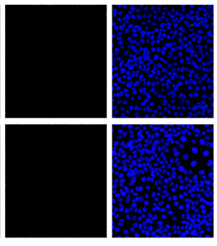

\section{)} os
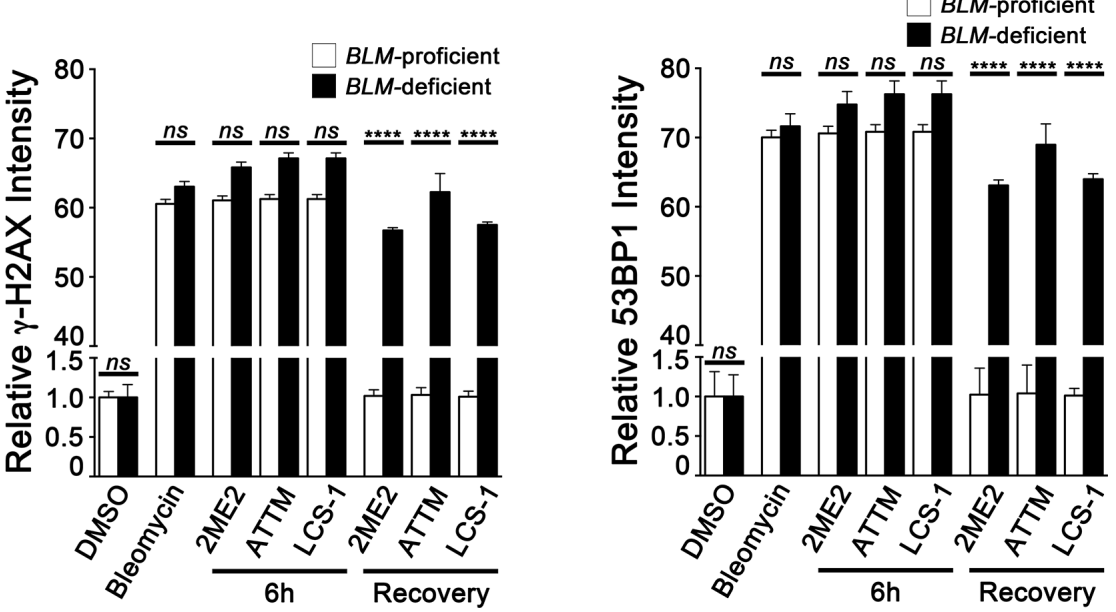

Figure 4: DNA DSBs persist in BLM-deficient cells treated with 2ME2, ATTM and LCS-1. A. Representative low-resolution $(10 \times)$ images presenting the qualitative changes in $\gamma$ - $\mathrm{H} 2 \mathrm{AX}$ and 53BP1 signal intensities within control (left) and $B L M$-deficient cells (right) treated with DMSO, bleomycin (positive control), 2ME2, ATTM, or LCS-1. Cells were imaged after $2 \mathrm{~h}(\mathrm{t}=2 \mathrm{~h}$; bleomycin) or $6 \mathrm{~h}(\mathrm{t}=6 \mathrm{~h}$; DMSO, 2ME2, ATTM and LCS-1) treatments, or following treatment, washout and a $36 \mathrm{~h}$ recovery phase $(\mathrm{t}=42 \mathrm{~h})$. Nuclei were counterstained with Hoechst, and images were acquired using identical exposure times at each wavelength so that qualitative and quantitative analyses could be performed. Hoechst, $\gamma-\mathrm{H} 2 \mathrm{AX}$ and 53BP1 are pseudo-colored blue, green, and red, respectively, within the merged images. Scale bars represent $100 \mu \mathrm{m}$. Note the persistence of $\gamma$-H2AX and 53BP1 signal intensities within the $B L M$-deficient cells following washout and recovery relative to controls. B. Graphs presenting the mean normalized $\gamma$-H2AX (left) and 53BP1 (right) signal intensities ( $\pm \mathrm{SD}$ ) within control and $B L M$-deficient cells treated with DMSO, bleomycin, 2ME2, ATTM, or LCS-1 or following washout and a $36 \mathrm{~h}$ recovery phase $(\mathrm{t}=42 \mathrm{~h})$. All data are presented relative to the DMSO-treated controls. Raw, unprocessed images were used to determine $\gamma$-H2AX and 53BP1 signal intensities. Note the persistence and statistically significant differences observed for $\gamma-\mathrm{H} 2 \mathrm{AX}$ and 53BP1 following washout and recovery within the $B L M$-deficient cells relative to controls (ns, not significant; ****, $p$-value $<0.0001$ ). 


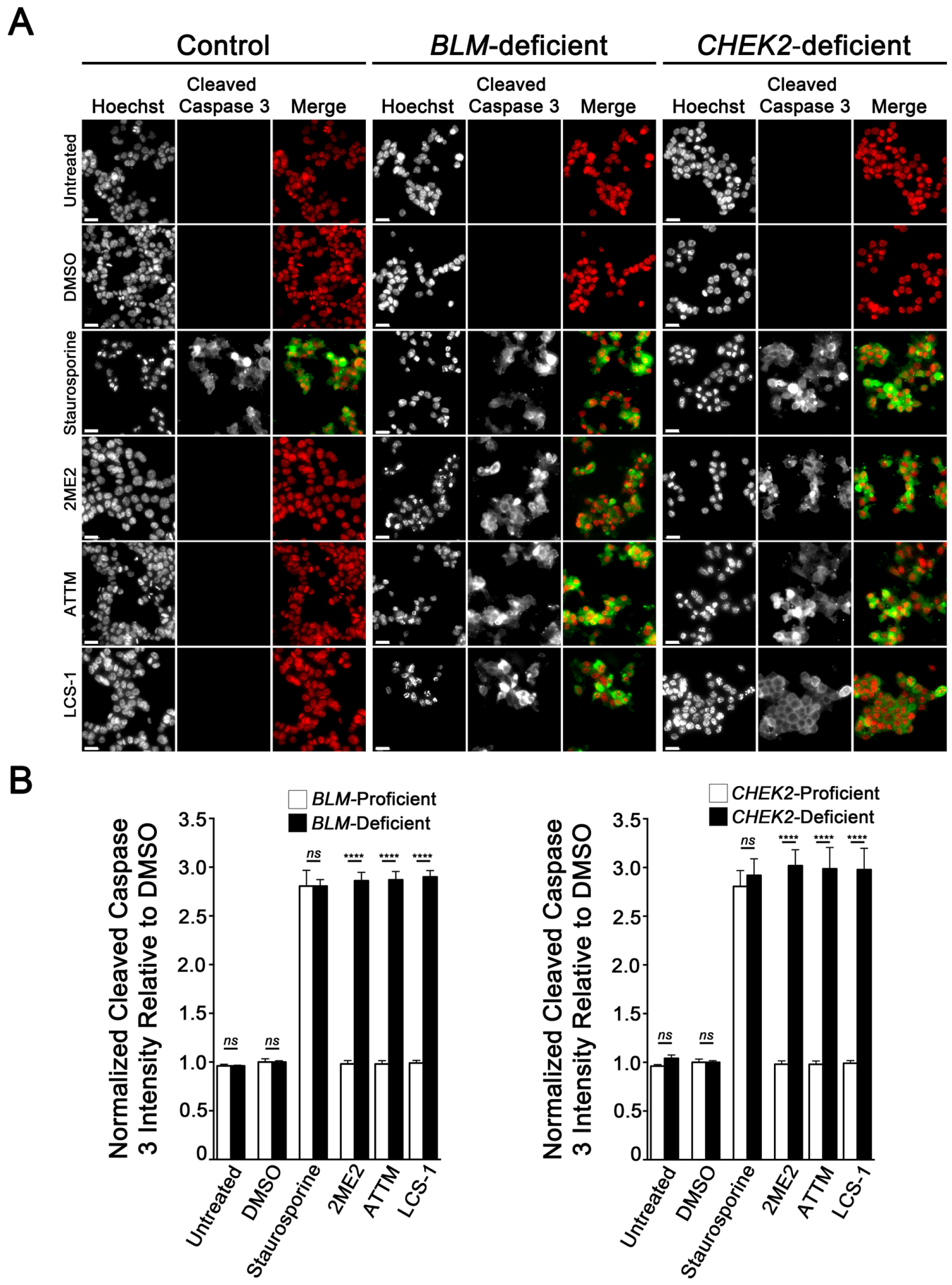

Figure 5: 2ME2, ATTM and LCS-1 induce apoptosis in $B L M$ - and $C H E K 2$-deficient cells. A. Representative low-resolution images $(10 \times)$ presenting the qualitative differences in cleaved Caspase 3 signal intensities within control, $B L M$-and $C H E K 2$-deficient cells treated with DMSO, staurosporine (positive control), 2ME2, ATTM and LCS-1. Cells were labeled for cleaved Caspase 3, while nuclei were counterstained with Hoechst. All images were collected using identical exposure times at each wavelength so that qualitative and quantitative analyses could be performed. Hoechst and cleaved Caspase 3 are pseudo-colored red and green, respectively within the merged images. Scale bars represent $30 \mu \mathrm{m}$. Note the visually striking increases in cleaved Caspase 3 signal intensities within the $B L M$ - and CHEK2-deficient cells treated with 2ME2, ATTM and LCS-1 relative to controls. B. Bar graphs depicting the mean normalized cleaved Caspase 3 signal intensities ( $\pm \mathrm{SD}$ ) within control, BLM- (left) and CHEK2-deficient (right) cells treated with DMSO, staurosporine, 2ME2, ATTM and LCS-1. All data are presented relative to DMSO treated controls. Cleaved Caspase 3 signal intensities were determined from raw, unprocessed images. Note the statistically significant increase in cleaved Caspase 3 signal intensities within the BLM-and CHEK2deficient cells relative to controls (ns, not significant; ****, $p$-value $<0.0001)$. 
were performed. First, standard colony formation assays were conducted 28-days in which DMSO, 2ME2, ATTM or LCS-1 was supplemented into growth media that was replaced every 2-days. In agreement with the above findings, statistically significant decreases were observed for the total number of $B L M$ - and CHEK2-deficient colonies treated with 2ME2, ATTM and LCS-1 relative to controls (Figure 6A). Next, the efficacy of the compounds was evaluated in 3D tumor sphere models. Tumor spheres were generated (see Materials and Methods) and treated with compounds or vehicle control, with media (with and without compounds) replaced every 2 days. Following a 14-day incubation period, nuclei were counterstained, tumor spheres were imaged and the sizes (diameters) were determined. In general, there was a statistically significant and $\sim 10$-fold decrease in the relative size of all BLM- and CHEK2-deficient tumor spheres treated with 2ME2, ATTM and LCS-1 relative to controls (Figure 6B). Collectively, these data indicate that 2ME2, ATTM, and LCS-1 treatments decrease the total number of $B L M$ - and $C H E K 2$-deficient colonies, and also the sizes of 3D tumor spheres, suggesting each is a strong lead candidate therapeutic compound.
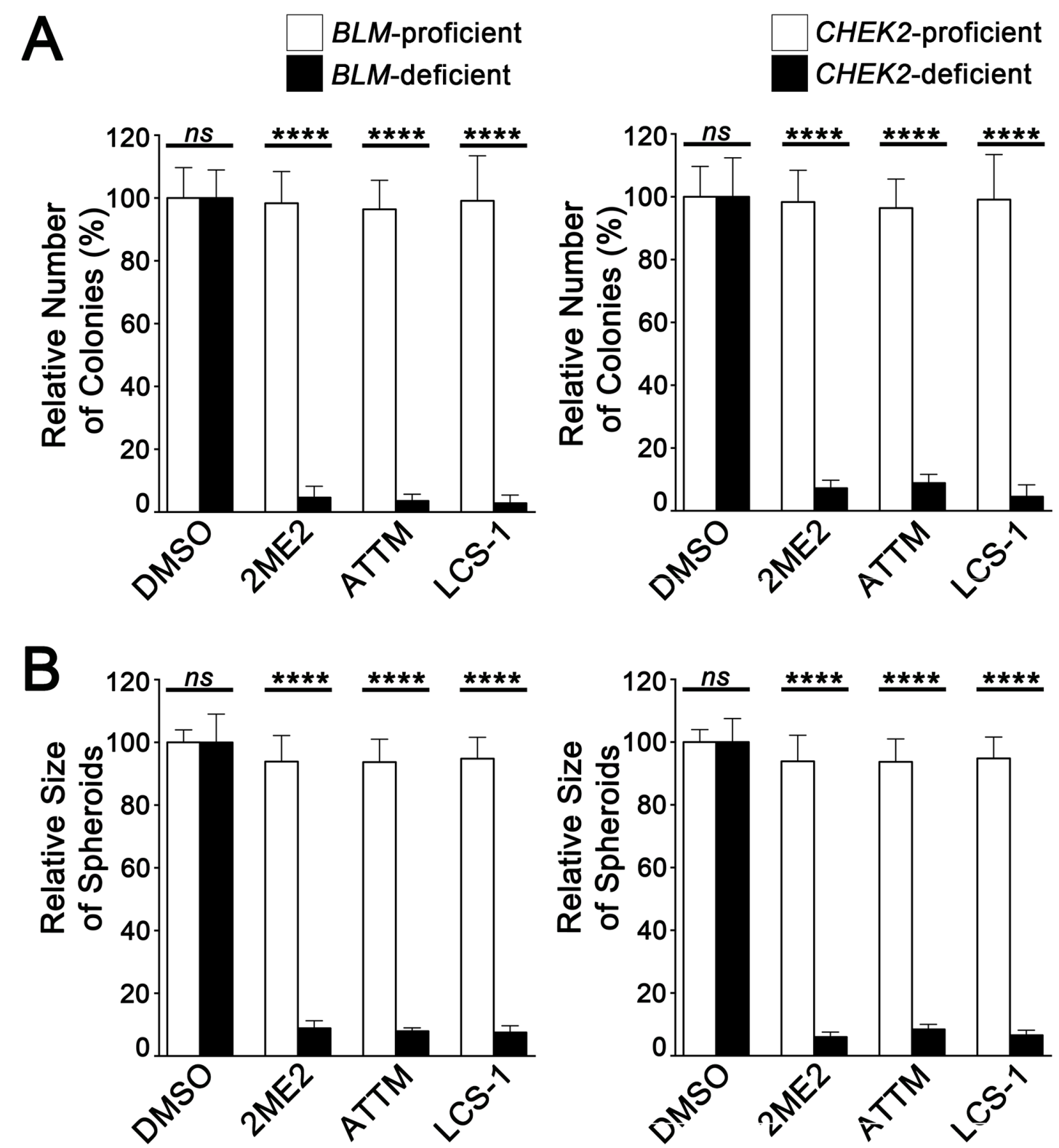

Figure 6: 2ME2, ATTM and LCS-1 inhibit growth of 2D and 3D cultures of BLM- and CHEK2-deficient cells. A. Bar graphs depicting statistically significant decreases in the mean number of BLM- and CHEK2-deficient colonies following 2ME2, ATTM and LCS-1 treatments relative to controls (ns, not significant; ****, $p$-value $<0.0001$ ). Cells were treated for 28 -days and the data are presented relative to DMSO treated controls $( \pm \mathrm{SD})$. B. Bar graphs presenting statistically significant decreases in the size of $B L M-$ and CHEK2-deficient 3D tumor spheres treated with 2ME2, ATTM and LCS-1 relative to controls (ns, not significant; $* * * *, p$-value $<0.0001$ ). All spheres were treated for 14-days and the data are presented relative to DMSO treated controls $( \pm$ SD). 


\section{DISCUSSION}

In this study, we evaluated the ability of SL interactions initially identified in yeast to predict evolutionarily conserved interactions within a human cell context. Specifically, we sought to determine the ability of SOD1 silencing and inhibition to exploit defects within two genes, BLM and CHEK2, which normally encode functions within the HDR pathway. Using two isogenic cell models we demonstrate that SOD1 silencing induces preferential killing within BLM- and CHEK2-deficient cells, and show that these SL interactions are conserved in an unrelated and immortalized cell type. We further show that three chemicals predicted to functionally substitute for SOD1 silencing also induce preferential killing within BLM- and CHEK2-deficient cells in short (5-day), moderate (14-day) and long-term (28-days) assays in both 2D culture and 3D tumor models. Finally, we show that each compound induces ROS, is associated with increases in DNA DSBs, and correlates with increases in apoptosis within BLM- and CHEK2-deficient cells. Accordingly, this study identifies two evolutionarily conserved SL interactions, namely BLM SOD1 and $C H E K 2$ SOD1, and defines SOD1 as a novel candidate drug target in cancers harboring $B L M$ and $C H E K 2$ defects. Finally, our data identify 2ME2, ATTM and LCS-1 as lead candidate compounds warranting further pre-clinical study. Collectively, this study underscores the utility of SL datasets generated in model systems (e.g. budding yeast) to uncover evolutionarily conserved and cancer-relevant interactions that will assist in cancer drug target discovery.

SOD1 is highly conserved throughout evolution [33] and its central role in the removal of superoxide radicals and the prevention of excessive oxidative DNA damage is well established in model organisms and humans $[34,35]$. SOD1 is a non-essential gene in yeast [36] and mice [37], and the transient nature of treatments is predicted to have minimal impact on normal human cells. Moreover, the $\mathrm{EC}_{50}$ values employed in this study are specific to the $B L M$ - and CHEK2-deficient cells, and are significantly lower than those of the controls (54- to 3, 150-fold). With respect to the compounds, ATTM has been employed for unrelated pathologies, while 2ME2 and LCS-1 remain relatively unexplored. For example, ATTM was originally employed to treat copper poisoning in livestock [38], while in humans it is commonly used to treat Wilson's disease, a neuropsychiatric disorder resulting from $\mathrm{Cu}^{2+}$ accumulation. Most recently, ATTM is under investigation for its anti-angiogenic potential based on its ability to prevent endothelial cell homing, cell motility and invasiveness [39-43]. In 2011, Somwar et al [21] identified LCS-1 as a selective SOD1 inhibitor and showed it induced killing in lung cancer cells. Most recently, several studies have begun to explore the susceptibility of vascular endothelial cells to ROS [44-47], suggesting SOD1 inhibitors and ROS inducers may hold additional potential as anti-angiogenic agents. In the current study, we repurposed these chemicals to induce SL killing within two specific genetic contexts (i.e. somatic BLMor CHEK2-deficiencies). Thus, it is possible that 2ME2, ATTM or LCS-1 treatments in BLM- or CHEK2-deficient/ defective tumors may induce anti-angiogenic effects that will synergize with the SL interactions to prevent tumor vascularization while inducing SL killing.

Although the primary goal of this work is to exploit somatic mutations in $B L M$ and $C H E K 2$, it is possible that 2ME2, ATTM and LCS-1 may also be effective in familial cancers, in much the same manner that PARP1 inhibitors are being evaluated in the context of familial breast and ovarian cancers harboring inherited defects in $B R C A 1 / 2$. In a familial cancer context, all individuals are expected to inherit a single wile-type allele and a mutant allele, and it is the subsequent loss of heterozygosity that contributes to the development of familial tumors. Thus, it may be possible to selectively target familial cancers as the non-cancerous (i.e. normal) cells are expected to harbor a single wild-type copy of $B L M$ or CHEK2, and produce sufficient protein to render these cells resistant to a SL attack.

Over the five past years, there have been numerous successful approaches designed to identify SL interactors of cancer-associated genes, with the ultimate goal of identifying novel drug targets. In 2013, Vizeacoumar et al [48] performed a genome-wide screen to uncover negative genetic interactions (i.e. SL interactors) across a set of isogenic cancer cell lines, including the $B L M$ deficient cells employed in the current study. Interestingly, they did not identify SOD1 as a lead SL interactor, which we attribute to two fundamental differences between our studies. First, Vizeacoumar and colleagues employed a microarray-based approach that is based on the loss of the bar-coded shRNAs within a population of cells, which may not have occurred within the timeframe of their experiment. Second, and perhaps most likely, although SOD1-induced killing is robust within our study, it may not have fallen below the operational threshold required for subsequent validation within their study. Nevertheless, our data complement their findings, and expands the number of validated SL interactors and drug targets of $B L M$-deficient cells.

The current study provides the first evidence that the dun1 sod1 SL interaction first identified in yeast [27], is evolutionarily conserved in a human cancer context. More specifically, we identify SOD1 as the first SL interactor and drug target capable of exploiting genetic defects in CHEK2. CHEK2 is a particularly attractive and unexplored gene to examine in direct SL tests as it has an established role in HDR [49], it is normally required for chromosome stability [50,51], and mutations are associated with both familial and sporadic cancers [52-55]. However, CHEK2 is also of interest as a therapeutic target that can potentiate the cytotoxic effects associated with DNA damage, 
radiation or chemotherapeutic compounds including camptothecin [56], PARP inhibitors or doxorubicin [57]. With the knowledge that CHEK2 regulates TP53mediated apoptosis [58], another therapeutic strategy has been to target CHEK2 activity to sensitize TP53deficient cells to compounds that induce genotoxic stress [59, 60]. In 2009, Jiang et al [60] demonstrated that following CHEK2 depletion, TP53-deficient cells were sensitized to doxorubicin, while the TP53-proficient cells were resistant. Although speculative, we predict that the simultaneous depletion of CHEK2 in combination with SOD1 silencing or 2ME2, ATTM or LCS-1 treatments may further exacerbate the sensitivity of TP53-deficient cells to doxorubicin. Thus, targeting SOD1 may have additional therapeutic implications beyond the $B L M$ and CHEK2 genetic contexts explored in the current study.

A major goal of the current study was to determine whether SOD1 was a shared SL interactor for genes involved in HDR in humans. Beyond the $B L M$ and CHEK2 contexts examined in the current study, SOD1 may represent a common therapeutic target capable of exploiting many additional genetic defects. Extensive SL data generated in budding yeast $[27,61,62]$ have shown that members of a given biological pathway (e.g. HDR) frequently share SL interactors. We previously showed that $R A D 54 B$, a gene that encodes a helicase functions within the HDR pathway is also SL with SOD1 [20]. While $R A D 54 B$ is somatically altered in $\sim 3.3 \%$ of CRCs $[3,4,6], B L M$ and $C H E K 2$ are mutated in up to $4.1 \%$ [6] and $6.9 \%[3,4,63]$, respectively. Thus, mutations in these three genes alone account for $\sim 14.4 \%$ of all CRCs, which amounts to $\sim 19,000$ Americans annually who may be potentially responsive to a SOD1-directed therapy. Due to the evolutionarily conserved nature of these SL interactions, it appears that SOD1 is a putative therapeutic hub that may harbor addition SL interactions with other HDR genes. Although speculative, this list could include other key HDR genes including ATM, BRCA1/2, MRE11, $R A D 50, N B S 1$ or $R A D 51$, which are also mutated in $\mathrm{CRC}$ and many other tumor types. Furthermore, somatic mutations of SOD1 are rare in cancer, and those few that do occur are mutually exclusive of those occurring for RAD54B, BLM and CHEK2 [3, 4, 6, 64-67]. These observations suggest that mutations leading to a loss of function for SOD1 are not tolerated in cancer cells harboring pre-existing mutations within $R A D 54 B, B L M$ or $C H E K 2$, and further support SOD1 as a strong candidate therapeutic target. Finally, SOD1 may also represent an attractive therapeutic target beyond CRC. Although RAD 54B, BLM and CHEK2 genes are mutated in CRC, they are also mutated in numerous additional cancers including pancreatic $(\sim 16.5 \%$ collectively $)$, prostate $(\sim 16.7 \%)$, melanoma $(\sim 15.1 \%)$ and endometrial ( $\sim 8.8 \%)$. Thus, studies aimed at exploring SOD1 as a candidate drug target in other HDR-defective gene contexts, and tumor types are highly warranted.

\section{ACKNOWLEDGMENTS}

We thank Drs. C.P. Case (University of Bristol, Bristol, UK) and B. Vogelstein (Johns Hopkins, Baltimore, MD) for providing cell lines, and Abcam Inc., for antibodies. We thank McManus laboratory members for helpful suggestions and critical review. We acknowledge the strong support of the Research Institute of Oncology and Hematology and the CancerCare Manitoba Foundation.

\section{GRANT SUPPORT/ FUNDING}

This work was funded by operating grants from the Canadian Institutes of Health Research (MOP-115179) and the CancerCare Manitoba Foundation.

\section{CONFLICTS OF INTEREST}

None.

\section{REFERENCES}

1. American Cancer Society (2015). Cancer Facts \& Figures 2015. Atlanta: American Cancer Society.

2. Sajesh BV, Guppy BJ, McManus KJ. Synthetic genetic targeting of genome instability in cancer. Cancers (Basel). 2013; 5:739-761.

3. Cerami E, Gao J, Dogrusoz U, Gross BE, Sumer SO, Aksoy BA, Jacobsen A, Byrne CJ, Heuer ML, Larsson E, Antipin Y, Reva B, Goldberg AP, Sander C, Schultz N. The cBio cancer genomics portal: an open platform for exploring multidimensional cancer genomics data. Cancer Discov. 2012; 2:401-404.

4. Gao J, Aksoy BA, Dogrusoz U, Dresdner G, Gross B, Sumer SO, Sun Y, Jacobsen A, Sinha R, Larsson E, Cerami E, Sander C, Schultz N. Integrative analysis of complex cancer genomics and clinical profiles using the cBioPortal. Sci Signal. 2013; 6:pl1.

5. Lengauer C, Kinzler KW, Vogelstein B. Genetic instabilities in human cancers. Nature. 1998; 396:643-649.

6. Cancer Genome Atlas N: Comprehensive molecular characterization of human colon and rectal cancer. Nature. 2012; 487:330-337.

7. Kitano K. Structural mechanisms of human RecQ helicases WRN and BLM. Frontiers in genetics. 2014; 5:366.

8. Zong C, Ji Y, He Q, Zhu S, Qin F, Tong J, Cao Y. Adaptive response in mice exposed to $900 \mathrm{MHZ}$ radiofrequency fields: Bleomycin-induced DNA and oxidative damage/ repair. Int J Radiat Biol. 2015; 91:270-276.

9. Daley JM, Chiba T, Xue X, Niu H, Sung P. Multifaceted role of the Topo IIIalpha-RMI1-RMI2 complex and DNA2 in the BLM-dependent pathway of DNA break end resection. Nucleic Acids Res. 2014; 42:11083-11091. 
10. Bohm S, Bernstein KA. The role of post-translational modifications in fine-tuning BLM helicase function during DNA repair. DNA Repair (Amst). 2014; 22:123-132.

11. Sturzenegger A, Burdova K, Kanagaraj R, Levikova M, Pinto C, Cejka P, Janscak P. DNA2 cooperates with the WRN and BLM RecQ helicases to mediate long-range DNA end resection in human cells. The Journal of biological chemistry. 2014; 289:27314-27326.

12. Bendtsen KM, Jensen MB, May A, Rasmussen LJ, Trusina A, Bohr VA, Jensen MH. Dynamics of the DNA repair proteins WRN and BLM in the nucleoplasm and nucleoli. Eur Biophys J. 2014; 43:509-516.

13. Manthei KA, Keck JL. The BLM dissolvasome in DNA replication and repair. Cellular and molecular life sciences : CMLS. 2013; 70:4067-4084.

14. Stolz A, Ertych N, Bastians H. Tumor suppressor CHK2: regulator of DNA damage response and mediator of chromosomal stability. Clinical Cancer Research : an official journal of the American Association for Cancer Research. 2011; 17:401-405.

15. Reinhardt HC, Jiang H, Hemann MT, Yaffe MB. Exploiting synthetic lethal interactions for targeted cancer therapy. Cell cycle. 2009; 8:3112-3119.

16. Bartek J, Bartkova J, Lukas J. DNA damage signalling guards against activated oncogenes and tumour progression. Oncogene. 2007; 26:7773-7779.

17. Jossen R, Bermejo R. The DNA damage checkpoint response to replication stress: A Game of Forks. Frontiers in genetics. 2013; 4:26.

18. Ciccia A, Elledge SJ. The DNA damage response: making it safe to play with knives. Molecular cell. 2010; 40:179-204.

19. Cogan N, Baird DM, Phillips R, Crompton LA, Caldwell MA, Rubio MA, Newson R, Lyng F, Case CP. DNA damaging bystander signalling from stem cells, cancer cells and fibroblasts after $\mathrm{Cr}(\mathrm{VI})$ exposure and its dependence on telomerase. Mutat Res. 2010; 683:1-8.

20. Sajesh BV, Bailey M, Lichtensztejn Z, Hieter P, McManus KJ. Synthetic lethal targeting of superoxide dismutase 1 selectively kills RAD54B-deficient colorectal cancer cells. Genetics. 2013; 195:757-767.

21. Somwar R, Erdjument-Bromage H, Larsson E, Shum D, Lockwood WW, Yang G, Sander C, Ouerfelli O, Tempst PJ, Djaballah H, Varmus HE. Superoxide dismutase 1 (SOD1) is a target for a small molecule identified in a screen for inhibitors of the growth of lung adenocarcinoma cell lines. Proceedings of the National Academy of Sciences of the United States of America. 2011; 108:16375-16380.

22. McManus KJ, Hendzel MJ. Using quantitative imaging microscopy to define the target substrate specificities of histone post-translational-modifying enzymes. Methods. 2005; 36:351-361.

23. Schneider CA, Rasband WS, Eliceiri KW. NIH Image to ImageJ: 25 years of image analysis. Nature methods. 2012; 9:671-675.
24. Chatr-Aryamontri A, Breitkreutz BJ, Oughtred R, Boucher L, Heinicke S, Chen D, Stark C, Breitkreutz A, Kolas N, O'Donnell L, Reguly T, Nixon J, Ramage L, Winter A, Sellam A, Chang C, et al. The BioGRID interaction database: 2015 update. Nucleic acids research. 2015; 43:D470-478.

25. Fridovich I. Superoxide radical and superoxide dismutases. Annual review of biochemistry. 1995; 64:97-112.

26. Juarez JC, Betancourt O Jr, Pirie-Shepherd SR, Guan X, Price ML, Shaw DE, Mazar AP, Donate F. Copper binding by tetrathiomolybdate attenuates angiogenesis and tumor cell proliferation through the inhibition of superoxide dismutase 1. Clinical Cancer Research : an official journal of the American Association for Cancer Research. 2006; 12:4974-4982.

27. Pan X, Ye P, Yuan DS, Wang X, Bader JS, Boeke JD. A DNA integrity network in the yeast Saccharomyces cerevisiae. Cell. 2006; 124:1069-1081.

28. McManus KJ, Barrett IJ, Nouhi Y, Hieter P. Specific synthetic lethal killing of RAD54B-deficient human colorectal cancer cells by FEN1 silencing. Proceedings of the National Academy of Sciences of the United States of America. 2009; 106:3276-3281.

29. Kachadourian R, Liochev SI, Cabelli DE, Patel MN, Fridovich I, Day BJ. 2-methoxyestradiol does not inhibit superoxide dismutase. Archives of biochemistry and biophysics. 2001; 392:349-353.

30. Cooke MS, Evans MD, Dizdaroglu M, Lunec J. Oxidative DNA damage: mechanisms, mutation, and disease. FASEB journal : official publication of the Federation of American Societies for Experimental Biology. 2003; 17:1195-1214.

31. Klaunig JE, Kamendulis LM, Hocevar BA. Oxidative stress and oxidative damage in carcinogenesis. Toxicologic pathology. 2010; 38:96-109.

32. Brierley DJ, Martin SA. Oxidative stress and the DNA mismatch repair pathway. Antioxid Redox Signal. 2013; 18:2420-2428.

33. Wang J, Xu G, Borchelt DR. Mapping superoxide dismutase 1 domains of non-native interaction: roles of intra- and intermolecular disulfide bonding in aggregation. Journal of neurochemistry. 2006; 96:1277-1288.

34. Corson LB, Strain JJ, Culotta VC, Cleveland DW. Chaperone-facilitated copper binding is a property common to several classes of familial amyotrophic lateral sclerosislinked superoxide dismutase mutants. Proceedings of the National Academy of Sciences of the United States of America. 1998; 95:6361-6366.

35. Reddi AR, Culotta VC. SOD1 integrates signals from oxygen and glucose to repress respiration. Cell. 2013; 152:224-235.

36. Chang EC, Crawford BF, Hong Z, Bilinski T, Kosman DJ. Genetic and biochemical characterization of $\mathrm{Cu}, \mathrm{Zn}$ superoxide dismutase mutants in Saccharomyces cerevisiae. The Journal of biological chemistry. 1991; 266:4417-4424. 
37. Kondo T, Reaume AG, Huang TT, Carlson E, Murakami K, Chen SF, Hoffman EK, Scott RW, Epstein CJ, Chan PH. Reduction of CuZn-superoxide dismutase activity exacerbates neuronal cell injury and edema formation after transient focal cerebral ischemia. The Journal of neuroscience : the official journal of the Society for Neuroscience. 1997; 17:4180-4189.

38. Suttle NF. Copper imbalances in ruminants and humans: unexpected common ground. Advances in nutrition. 2012; 3:666-674.

39. Pan Q, Kleer CG, van Golen KL, Irani J, Bottema KM, Bias C, De Carvalho M, Mesri EA, Robins DM, Dick RD, Brewer GJ, Merajver SD. Copper deficiency induced by tetrathiomolybdate suppresses tumor growth and angiogenesis. Cancer research. 2002; 62:4854-4859.

40. Henry NL, Dunn R, Merjaver S, Pan Q, Pienta KJ, Brewer G, Smith DC. Phase II trial of copper depletion with tetrathiomolybdate as an antiangiogenesis strategy in patients with hormone-refractory prostate cancer. Oncology. 2006; 71:168-175.

41. Gartner EM, Griffith KA, Pan Q, Brewer GJ, Henja GF, Merajver SD, Zalupski MM. A pilot trial of the antiangiogenic copper lowering agent tetrathiomolybdate in combination with irinotecan, 5-flurouracil, and leucovorin for metastatic colorectal cancer. Investigational new drugs. 2009; 27:159-165.

42. Kim KK, Lange TS, Singh RK, Brard L, Moore RG. Tetrathiomolybdate sensitizes ovarian cancer cells to anticancer drugs doxorubicin, fenretinide, 5-fluorouracil and mitomycin C. BMC cancer. 2012; 12:147.

43. Kumar P, Yadav A, Patel SN, Islam M, Pan Q, Merajver SD, Teknos TN. Tetrathiomolybdate inhibits head and neck cancer metastasis by decreasing tumor cell motility, invasiveness and by promoting tumor cell anoikis. Molecular cancer. 2010; 9:206.

44. Jerkic M, Letarte M. Contribution of oxidative stress to endothelial dysfunction in hereditary hemorrhagic telangiectasia. Frontiers in genetics. 2015; 6:34.

45. Munzel T, Gori T, Bruno RM, Taddei S. Is oxidative stress a therapeutic target in cardiovascular disease? European heart journal. 2010; 31:2741-2748.

46. Cimmino G, Cirillo P, Ragni M, Conte S, Uccello G, Golino P. Reactive oxygen species induce a procoagulant state in endothelial cells by inhibiting tissue factor pathway inhibitor. Journal of thrombosis and thrombolysis. 2015; 40:186-92.

47. Fernandes AS, Florido A, Saraiva N, Cerqueira S, Ramalhete S, Cipriano M, Cabral MF, Miranda JP, Castro M, Costa J, Oliveira NG. Role of the Copper(II) Complex $\mathrm{Cu}[15]$ pyN in Intracellular ROS and Breast Cancer Cell Motility and Invasion. Chemical biology \& drug design. 2015; .

48. Vizeacoumar FJ, Arnold R, Vizeacoumar FS, Chandrashekhar M, Buzina A, Young JT, Kwan JH,
Sayad A, Mero P, Lawo S, Tanaka H, Brown KR, Baryshnikova A, Mak AB, Fedyshyn Y, Wang Y, et al. A negative genetic interaction map in isogenic cancer cell lines reveals cancer cell vulnerabilities. Molecular systems biology. 2013; 9:696.

49. Smith J, Tho LM, Xu N, Gillespie DA. The ATM-Chk2 and ATR-Chk1 pathways in DNA damage signaling and cancer. Advances in cancer research. 2010; 108:73-112.

50. Petsalaki E, Zachos G. Chk2 prevents mitotic exit when the majority of kinetochores are unattached. The Journal of cell biology. 2014; 205:339-356.

51. Stolz A, Ertych N, Bastians H. Loss of the tumoursuppressor genes $\mathrm{CHK} 2$ and BRCA1 results in chromosomal instability. Biochemical Society transactions. 2010; 38:1704-1708.

52. Kleibl Z, Havranek O, Hlavata I, Novotny J, Sevcik J, Pohlreich P, Soucek P. The CHEK2 gene I157T mutation and other alterations in its proximity increase the risk of sporadic colorectal cancer in the Czech population. European journal of cancer. 2009; 45:618-624.

53. Ow GS, Ivshina AV, Fuentes G, Kuznetsov VA. Identification of two poorly prognosed ovarian carcinoma subtypes associated with CHEK2 germ-line mutation and non-CHEK2 somatic mutation gene signatures. Cell cycle. 2014; 13:2262-2280.

54. Bell DW, Kim SH, Godwin AK, Schiripo TA, Harris PL, Haserlat SM, Wahrer DC, Haiman CA, Daly MB, Niendorf KB, Smith MR, Sgroi DC, Garber JE, Olopade OI, Le Marchand L, Henderson BE, et al. Genetic and functional analysis of CHEK2 (CHK2) variants in multiethnic cohorts. International journal of cancer Journal international du cancer. 2007; 121:2661-2667.

55. Marsh A, Healey S, Lewis A, Spurdle AB, Kedda MA, Khanna KK, kConFab , Mann GJ, Pupo GM, Lakhani SR, Chenevix-Trench G. Mutation analysis of five candidate genes in familial breast cancer. Breast cancer research and treatment. 2007; 105:377-389.

56. Jobson AG, Lountos GT, Lorenzi PL, Llamas J, Connelly J, Cerna D, Tropea JE, Onda A, Zoppoli G, Kondapaka S, Zhang G, Caplen NJ, Cardellina JH 2nd, Yoo SS, Monks A, Self C, et al. Cellular inhibition of checkpoint kinase 2 (Chk2) and potentiation of camptothecins and radiation by the novel Chk2 inhibitor PV1019 [7-nitro1H-indole-2-carboxylic acid \{4-[1-(guanidinohydrazone)ethyl]-phenyl $\}$-amide]. The Journal of pharmacology and experimental therapeutics. 2009; 331:816-826.

57. Anderson VE, Walton MI, Eve PD, Boxall KJ, Antoni L, Caldwell JJ, Aherne W, Pearl LH, Oliver AW, Collins I, Garrett MD. CCT241533 is a potent and selective inhibitor of CHK2 that potentiates the cytotoxicity of PARP inhibitors. Cancer research. 2011; 71:463-472.

58. Zhou BB, Bartek J. Targeting the checkpoint kinases: chemosensitization versus chemoprotection. Nature reviews Cancer. 2004; 4:216-225. 
59. Morandell S, Yaffe MB. Exploiting synthetic lethal interactions between DNA damage signaling, checkpoint control, and p53 for targeted cancer therapy. Progress in molecular biology and translational science. 2012; 110:289-314.

60. Jiang $\mathrm{H}$, Reinhardt HC, Bartkova J, Tommiska J, Blomqvist C, Nevanlinna H, Bartek J, Yaffe MB, Hemann MT. The combined status of ATM and p53 link tumor development with therapeutic response. Genes \& development. 2009; 23:1895-1909.

61. Stark C, Breitkreutz BJ, Reguly T, Boucher L, Breitkreutz A, Tyers M. BioGRID: a general repository for interaction datasets. Nucleic acids research. 2006; 34:D535-539.

62. Chatr-Aryamontri A, Breitkreutz BJ, Heinicke S, Boucher L, Winter A, Stark C, Nixon J, Ramage L, Kolas N, O'Donnell L, Reguly T, Breitkreutz A, Sellam A, Chen D, Chang C, Rust J, et al. The BioGRID interaction database: 2013 update. Nucleic acids research. 2013; 41:D816-823.

63. Seshagiri S, Stawiski EW, Durinck S, Modrusan Z, Storm EE, Conboy CB, Chaudhuri S, Guan Y,
Janakiraman V, Jaiswal BS, Guillory J, Ha C, Dijkgraaf GJ, Stinson J, Gnad F, Huntley MA, et al. Recurrent R-spondin fusions in colon cancer. Nature. 2012; 488:660-664.

64. Cancer Genome Atlas Research Network: Integrated genomic analyses of ovarian carcinoma. Nature. 2011; 474:609-615.

65. Cancer Genome Atlas Research Network: Comprehensive genomic characterization defines human glioblastoma genes and core pathways. Nature. 2008; 455:1061-1068.

66. Cancer Genome Atlas Research Network: Comprehensive genomic characterization of squamous cell lung cancers. Nature. 2012; 489:519-525.

67. Cancer Genome Atlas Research Network: Comprehensive molecular portraits of human breast tumours. Nature. 2012; 490:61-70. 